\title{
Macroscopic behavior of polar nematic gels and elastomers
}

\author{
Helmut R. Brand ${ }^{1,2, a}$, Harald Pleiner ${ }^{2, b}$, and Daniel Svenšek ${ }^{3}$ \\ 1 Theoretische Physik III, Universität Bayreuth, 95440 Bayreuth, Germany \\ 2 Max-Planck-Institute for Polymer Research, POBox 3148, 55021 Mainz, Germany \\ 3 Department of Physics, Faculty of Mathematics and Physics, University of Ljubljana, 1000 Ljubljana, Slovenia
}

Received 30 May 2016 and Received in final form 5 October 2016

Published online: 10 November 2016

(c) The Author(s) 2016. This article is published with open access at Springerlink.com

\begin{abstract}
We present the derivation of the macroscopic equations for uniaxial polar nematic gels and elastomers. We include the strain field as well as relative rotations as independent dynamic macroscopic degrees of freedom. As a consequence, special emphasis is laid on possible static and dynamic cross-couplings between these macroscopic degrees of freedom associated with the network, and the other macroscopic degrees of freedom including reorientations of the macroscopic polarization. In particular, we find static and dissipative dynamic cross-couplings between strain fields and relative rotations on one hand and the macroscopic polarization on the other that allow for new possibilities to manipulate polar nematics. To give one example each for the effects of a static and a dissipative cross-coupling: we find that a static electric field applied perpendicularly to the polar preferred direction leads to relative rotations while dynamically relative rotations can lead to transverse electric currents. In addition to a permanent network, we also consider the effect of a transient network, which is particularly important for the case of gels, melts and concentrated polymer solutions. A section on the influence of macroscopic chirality is included as well.
\end{abstract}

\section{Introduction}

An open key issue in the field of complex fluids is the question to what extent fluidity is compatible with static macroscopic polar order in three dimensions. About three decades ago there were some early experimental efforts along these lines for nematic [1] and pyramidic [2] low molecular weight liquid crystals. These early experimental investigations triggered a theoretical study of GinzburgLandau type for polar nematics, where it was shown that spontaneous splay phases should play an important role in such systems [3]. More recently liquid crystalline phases formed by bent-core molecules were predicted [4] and shown experimentally $[5,6]$ to have polar directions for smectic liquid crystalline phases. After that liquid crystalline phases formed by bent-core molecules were studied theoretically and experimentally from various aspects (compare, for example, refs. [7-9]), but reports of nematic phases formed by bent-core molecules remained scarce $[10-14]$. In parallel it was pointed out on the basis of a symmetry analysis that for biaxial nematic phases, which are fluid in three dimensions, there are several pos-

\footnotetext{
a e-mail: brand@uni-bayreuth.de

b e-mail: pleiner@mpip-mainz.mpg.de
}

sibilities to have biaxial nematic phases with polar order [15]. From an experimental point of view there was progress in this direction in the field of main chain liquid crystalline polymers such as polypeptides, polyesters, and Vectra for which Watanabe's group showed [16-18] that one can have nematic phases with polar order including biaxial polar nematic phases with $C_{1 h}$ symmetry.

Clearly a macroscopic dynamic description of polar nematics will play an important role in elucidating, characterizing and suggesting new experiments for this class of materials. We use the term macroscopic dynamics to describe the low frequency long wavelength behavior of a type of material. In addition to the classical hydrodynamic variables, namely the conserved quantities and the variables connected to spontaneously broken continuous symmetries [19-21], one also incorporates so-called macroscopic variables [21], which relax on a finite, but sufficiently long time scale to become important for the macroscopic behavior of a given system. This concept has been introduced by Khalatnikov for the superfluid order parameter near the normal-fluid-superfluid $\lambda$-transition in ${ }^{4} \mathrm{He}[22]$ and has since been applied to many different systems including the superfluid phases of ${ }^{3} \mathrm{He}[23,24]$ and phase transitions in liquid crystals $[25,26]$. 
In the framework of macroscopic dynamics low molecular weight polar nematic liquid crystals were studied for the case without external electric fields in [27]. On the other hand, (ordinary) nematic gels and elastomers are well investigated [28]. Here we study polar nematic gels and elastomers. In these systems the preferred direction is polar, in contrast to ordinary nematic ones where a director exists. In addition, we include isotropic gels and elastomers that do not provide preferred directions by their own. Since the polar direction is the only preferred direction, the system is uniaxial. The polar and elastic aspects of the system can be realized by two different subsystems, e.g. by a polymeric elastomer swollen by a polar low molecular weight nematic, or by a single system, e.g. a cross-linked polymer with polar side-chain order. The static and dynamic interactions between the two aspects of such a system are a main topic of the present study.

In addition to the usual conserved quantities we have as macroscopic variables the variations of the polar preferred direction, associated with the spontaneously broken rotational symmetry, and the degree of polar orientational order. The network brings along as additional macroscopic variables the strain tensor as well as relative rotations between the polar order and the network. This is in fact the first occasion that relative rotations, a concept pioneered by de Gennes [29] for nematic liquid crystalline elastomers, is taken into account for systems with static polar order. The polar preferred direction, which changes sign under the parity operation, is an important ingredient for the generation of cross-coupling terms. We also analyze the influence of external electric fields. The influence of a transient network, an important concept for polymeric and elastomeric systems [30,31], is investigated as well. We will show that transient elasticity, a macroscopic approach, which incorporates transient networks systematically into hydrodynamics [32-40], will lead to additional coupling terms to lowest order in the wave vector. In addition we study the influence of chirality on the macroscopic behavior of polar cholesterics as well as polar cholesteric gels and elastomers. This appears to be the first class of condensed matter systems described macroscopically for which one has two quantities breaking parity symmetry, namely a polar preferred direction as well as a pseudoscalar quantity $q_{0}$ associated with macroscopic chirality.

We concentrate on bulk hydrodynamics and will not systematically discuss boundary conditions. In the absence of external fields boundary conditions for the orientation of the polarization are important to suppress a possible polar splay phase. They also are crucial when discussing defects and textures. This is an interesting topic for complex systems as the present one, but is beyond the scope of this manuscript, where a homogeneous ground state is considered.

The present paper is organized as follows. In sect. 2 we describe the choice of the macroscopic variables, the statics and the thermodynamics. In sect. 3 we derive the resulting dynamic macroscopic equations for the case of a permanent network. In sect. 4 we investigate some simple solutions of the macroscopic equations presented. In sect. 5 we analyze how a transient network changes the macroscopic behavior and in sect. 6 the influence of macroscopic chirality is discussed. Finally we present in sect. 7 a brief summary and conclusions. In appendix A we give a Ginzburg-Landau-type analysis of the isotropicferroelectric phase transition in polar nematic gels and elastomers thus generalizing earlier work done for low molecular weight liquid crystals [3]. This also defines the ground state whose hydrodynamics we are describing.

\section{Derivation of macroscopic equations}

\subsection{Hydrodynamic and macroscopic variables}

To derive macroscopic equations for polar nematic gels and elastomers we generalize suitably the macroscopic dynamics of polar nematics derived in ref. [27]. According to the Eulerian description all variables are local fields, i.e. volume densities that depend on space and time.

In a next step we must clarify, which type of polar nematic gels and elastomers we want to study in the following. In ref. [15] we have shown that - depending on the number of polar and non-polar directions - there can be, on the basis of symmetry considerations, a fairly large number of biaxial nematic phases. Here we focus on the simplest possibility of a polar nematic gel: we assume that there is one preferred direction associated with polar order. Thus one has overall uniaxial symmetry. We characterize the polar order with the macroscopic polarization $\boldsymbol{P}$, which can be decomposed into the unit vector $\hat{\boldsymbol{p}}$ and the modulus $P=|\boldsymbol{P}|$. Thus, the relevant variables [21] come in three classes.

The first class of variables, the conserved quantities, contains those already known from a simple fluid, the mass density $\rho$, the energy density $\varepsilon$ and the momentum density $\boldsymbol{g}$. In our case we add another variable, the concentration of the polar particles $(c)$. In lyotropic systems one could also take into account additionally the concentration of the solvent $c_{S}$ without changing the major results, since it has the same transformation behavior as $c$ and thus makes the same type of coupling terms.

In the second class we have the variables that are related to spontaneously broken continuous symmetries. In our case we have the orientation of the macroscopic polarization, $\hat{\boldsymbol{p}}$, which is associated with spontaneously broken rotational symmetry. The variations of $\hat{\boldsymbol{p}}, \delta \hat{p}_{i}$, with $\hat{p}_{i} \cdot \delta \hat{p}_{i}=0$ in first order, are truly hydrodynamic. $\hat{\boldsymbol{p}}$ is a polar vector and thus odd under parity and even under time reversal; the former property leads to a number of static and dynamic cross-coupling terms unknown from conventional uniaxial nematics. The modulus or magnitude of the macroscopic polarization, $P$, belongs to the third class of variables, which relax on a long, but finite time scale. The main difference to ordinary nematics lies in the fact that $\hat{p}_{i}$ is a true vector (no general $\hat{p}_{i} \rightarrow-\hat{p}_{i}$ invariance, but $\hat{p}_{i} \rightarrow-\hat{p}_{i}$ under spatial inversion) and in 
the modulus variable $P$, which is strongly susceptible to electric fields, in contrast to the nematic order parameter modulus. It should be noted that even in ordinary nematics the modulus $(S)$ has been treated as an additional degree of freedom $[41,42]$, although it is only weakly susceptible to external fields and its fluctuations often have a rather short relaxation time. Due to the strong coupling of $P$ to electric fields, it is clearly very important to keep this variable in polar nematics.

The presence of a network gives rise to the strain tensor $u_{i j}$ as a macroscopic variable; in its linearized version it reads $u_{i j}=\frac{1}{2}\left(\nabla_{i} u_{j}+\nabla_{j} u_{i}\right)$ with the displacement field $u_{i}$. Due to the simultaneous presence of a network as well as of the variables $\delta \hat{p}_{i}$, relative rotations $\tilde{\Omega}_{i}$ as pioneered by de Gennes [29] for nematic elastomers become an important macroscopic variable, which can be introduced via $\tilde{\Omega}_{i}=\delta \hat{p}_{i}-\Omega_{i}^{\perp}$ in analogy to the case of nematic liquid crystalline elastomers [28], where now $\Omega_{i}^{\perp}=\hat{p}_{j} \Omega_{i j}$ with $\Omega_{i j}=\frac{1}{2}\left(\nabla_{i} u_{j}-\nabla_{i} u_{i}\right)$ and $\hat{p}_{i} \Omega_{i}^{\perp}=0$. This variable describes the fact that in the presence of (vector or) tensor fields, rotations of $\hat{p}_{i}$ do not cost energy, only if also those fields are rotated the same way, and cost energy otherwise. Relative rotations are not truly hydrodynamic variables, but relax slowly enough to be considered here.

Throughout this paper we stick to the splitting of $\boldsymbol{P}$ into its modulus and its orientation, because this reveals the different hydrodynamic nature of the latter variables and facilitates comparison with ordinary nematics. We stress that no additional (static and/or dynamic) material parameters or effects are introduced by this procedure and we have checked that using $\boldsymbol{P}$ as a variable leads to a completely equivalent macroscopic dynamics (for an analogous discussion for ordinary nematics compare ref. [38]). On the contrary, however, in a Ginzburg-Landau-type description of the phase transition from the isotropic (no $\boldsymbol{P}$ ) phase to a polar nematic gel or elastomer (finite $\boldsymbol{P}$ ) the vector $\boldsymbol{P}$ must be used as a variable. This is described and discussed in detail in appendix A.

In the next section we first give the standard thermodynamic relations among the various degrees of freedom ensuring a description compatible with basic thermodynamic principles. The static part of the hydrodynamics is then presented in form of a total energy expression describing how deviations from the thermodynamic ground state enhance the energy. Subsequently, new symbols are defined, the forms of the material tensors involved are given thereby defining the static susceptibilities, and finally the physical meaning and implications of various energy contributions are sketched.

\subsection{Statics and thermodynamics}

To derive the static properties of our system we formulate the local first law of thermodynamics relating changes in the entropy density $\sigma$ to changes in the hydrodynamic and macroscopic variables discussed above. We find the Gibbs relation $[43]$

$$
\begin{aligned}
\mathrm{d} \varepsilon= & T \mathrm{~d} \sigma+\mu \mathrm{d} \rho+\mu_{c} \mathrm{~d} c+v_{i} \mathrm{~d} g_{i}+E_{i} \mathrm{~d} D_{i} \\
& +h_{i}^{\prime} \mathrm{d} \hat{p}_{i}+h^{\prime P} \mathrm{~d} P+\Phi_{i j} \mathrm{~d} \nabla_{j} \hat{p}_{i}+\Phi_{i}^{P} \mathrm{~d} \nabla_{i} P \\
& +\psi_{i j} \mathrm{~d} u_{i j}+L_{i}^{\perp} \mathrm{d} \tilde{\Omega}_{i},
\end{aligned}
$$

where we have kept inhomogeneous variations of the polarization magnitude, $\nabla_{i} P$, which become relevant for defects as well as for inhomogeneous external fields. Similar to the case of an ordinary nematic director, homogeneous variations of the preferred direction $\hat{\boldsymbol{p}}$ do not cost energy due to the spontaneous nature of the broken rotational symmetry, except in the presence of an external (symmetry breaking) field; thus, in the field-free, homogeneous case $h_{i}^{\prime}=0$. In addition, $h_{i}^{\prime} \hat{p}_{i}=0$, since $\hat{p}_{i}$ is a unit vector.

In eq. (1) the thermodynamic quantities, temperature $T$, chemical potential $\mu$, relative chemical potential $\mu_{c}$, electric field $E_{i}$, velocity $v_{i}$, the elastic stress $\psi_{i j}$, the "relative molecular field" $L_{i}^{\perp}$ associated with relative rotations, the molecular fields associated with the polarization $h^{\prime P}$, $\Phi_{i}^{P}, h_{i}^{\prime}$, and $\Phi_{i j}$ are defined as partial derivatives of the energy density with respect to the appropriate variables [21]. If we neglect surface effects and integrate eq. (1) by parts we can obtain a simplified expression for the Gibbs relation

$$
\begin{aligned}
\mathrm{d} \varepsilon= & T \mathrm{~d} \sigma+\mu \mathrm{d} \rho+\mu_{c} \mathrm{~d} c+v_{i} \mathrm{~d} g_{i}+E_{i} \mathrm{~d} D_{i} \\
& +W \mathrm{~d} P+h_{i}^{p} \mathrm{~d} \hat{p}_{i}+\psi_{i j} \mathrm{~d} u_{i j}+L_{i}^{\perp} \mathrm{d} \tilde{\Omega}_{i},
\end{aligned}
$$

where the molecular fields $h^{P}$ and $h_{i}^{p}$ are given by $W=$ $h^{\prime P}-\nabla_{j} \Phi_{j}^{P}$ and $h_{i}^{p}=h_{i}^{\prime}-\nabla_{j} \Phi_{i j}$, respectively.

In the equilibrium state considered here, the polarization magnitude, $P_{0}$, is constant and a given material parameter. The orientation of the polarization, $\hat{p}_{i}^{0}$ is homogeneous and arbitrary. Thus, we assume that external fields or boundary conditions suppress a splay structure as ground state, possible for polar systems, which anyhow cannot exist defect-free in the whole space. In an external electric field $\boldsymbol{E}$, the equilibrium polarization $\boldsymbol{P}_{0}$ is along $\boldsymbol{E}$ and $P_{0}$ is in addition also a function of the field strength, $P_{0}=P_{0}(E)$, a function which we will not specify further and which we assume to be known and which we will still call $P_{0}$. Due to electrostriction a finite polarization leads to deformations of the network, which we incorporate already in the ground state (cf. the discussion of eq. (11)). For an extended discussion of the ground state cf. appendix A.

Taking this equilibrium state as the stable ground state, the energy density expanded in all variables about this state has to be convex. In addition, this energy density must be invariant under time reversal as well as under parity and it must be invariant under rigid rotations, rigid translations and covariant under Galilei transformations. Taking into account these symmetry arguments we get, for deviations of the variables from the ground state in 
harmonic approximation

$$
\begin{aligned}
\varepsilon= & \varepsilon_{0}+\frac{1}{2} P_{0} E\left(\delta \hat{p}_{i}\right)^{2}+\frac{\alpha}{2}(\delta P)^{2}+\frac{1}{2} L_{i j}\left(\nabla_{i} P\right)\left(\nabla_{j} P\right) \\
& +\frac{1}{2} K_{i j k l}\left(\nabla_{i} \hat{p}_{j}\right)\left(\nabla_{k} \hat{p}_{l}\right)+M_{i j k}\left(\nabla_{i} P\right)\left(\nabla_{j} \hat{p}_{k}\right) \\
& +\left(\gamma_{1} \delta \rho+\gamma_{2} \delta \sigma+\gamma_{3} \delta c\right) P_{0} \delta P \\
& +\left(\beta_{1} \delta \rho+\beta_{2} \delta \sigma+\beta_{3} \delta c\right) \hat{p}_{i} \nabla_{i} P \\
& +\left(\bar{\beta}_{1} \delta \rho+\bar{\beta}_{2} \delta \sigma+\bar{\beta}_{3} \delta c\right) \nabla_{i} \hat{p}_{i} \\
& +\frac{1}{2} c_{i j k l} u_{i j} u_{k l}+\frac{1}{2} D_{1} \tilde{\Omega}_{i} \tilde{\Omega}_{i}+\frac{1}{2} D_{2}\left(\delta_{i k}^{\perp} \hat{p}_{j}+\delta_{i j}^{\perp} \hat{p}_{k}\right) \tilde{\Omega}_{i} u_{j k} \\
& +\left(\chi_{i j}^{\rho} \delta \rho+\chi_{i j}^{\sigma} \delta \sigma+\chi_{i j}^{c} \delta c+\chi_{i j}^{P} P_{0} \delta P+\chi_{i j k}^{p} \delta \hat{p}_{k}\right) u_{i j} \\
& +\zeta_{i j k l} u_{i j} \nabla_{k} \hat{p}_{l}+\zeta_{i j k}^{P} u_{i j} \nabla_{k} P+\zeta_{i j k}^{E} u_{i j} E_{k} \\
& +\zeta_{i j}^{\Omega E} \tilde{\Omega}_{i} E_{j}+\zeta_{i j k}^{\Omega p} \tilde{\Omega}_{i} \nabla_{j} \hat{p}_{k}+\zeta_{i j}^{\Omega P} \tilde{\Omega}_{i} \nabla_{j} P \\
& +\zeta_{i j k}^{\mathrm{flexo}} E_{k} \nabla_{i} \hat{p}_{j},
\end{aligned}
$$

where $\varepsilon_{0}$ contains all the contributions characteristic of a miscible binary fluid mixture [21] and where $\delta$ denotes deviations from the equilibrium value, in particular $\delta P=$ $P-P_{0}, \delta \hat{p}_{i}=\hat{p}_{i}-\hat{p}_{i}^{0}, \delta c=c-c_{0}$ etc. The polarization electric coupling, $-\boldsymbol{P} \cdot \boldsymbol{E}$, translates into the hydrodynamic electric orientation energy using $E_{i}=E \hat{p}_{i}^{0}$ and $\hat{p}_{i}^{0} \delta \hat{p}_{i}=$ $-\frac{1}{2}\left(\delta \hat{p}_{i}\right)^{2}$ due to the requirement $\left(\hat{p}_{i}^{0}+\delta \hat{p}_{i}\right)^{2}=1$, and is therefore proportional to $P_{0}$ and $E$.

Due to the existence of the preferred direction $\hat{p}_{i}$, all material tensors reflect this uniaxiality, in particular

$$
\begin{aligned}
K_{i j k l}= & \frac{1}{2} K_{1}\left(\delta_{i j}^{\perp} \delta_{k l}^{\perp}+\delta_{i l}^{\perp} \delta_{j k}^{\perp}\right)+K_{2} \hat{p}_{r} \epsilon_{r i j} \hat{p}_{q} \epsilon_{q k l} \\
& +K_{3} \hat{p}_{k} \hat{p}_{i} \delta_{l j}^{\perp}, \\
L_{i j}= & K_{4} \hat{p}_{i} \hat{p}_{j}+K_{5} \delta_{i j}^{\perp}, \\
M_{i j k}= & K_{6}\left(\hat{p}_{i} \delta_{j k}^{\perp}+\hat{p}_{j} \delta_{i k}^{\perp}\right),
\end{aligned}
$$

where $\epsilon_{i j k}$ is the totally antisymmetric symbol and $\delta_{i j}^{\perp}$ the transverse Kronecker delta, $\delta_{i j}^{\perp}=\delta_{i j}-\hat{p}_{i} \hat{p}_{j}$. The material tensor $\zeta_{i j k l}$ takes the form

$$
\begin{aligned}
\zeta_{i j k l}= & \zeta_{1} \delta_{i j}^{\perp} \delta_{k l}^{\perp}+\zeta_{2} \delta_{k l}^{\perp} \hat{p}_{i} \hat{p}_{j}+\zeta_{3}\left(\delta_{i k}^{\perp} \delta_{j l}^{\perp}+\delta_{i l}^{\perp} \delta_{j k}^{\perp}\right) \\
& +\zeta_{4}\left(\delta_{i l}^{\perp} \hat{p}_{j} \hat{p}_{k}+\delta_{j l}^{\perp} \hat{p}_{i} \hat{p}_{k}\right),
\end{aligned}
$$

having only four coefficients due to the transversality of $\delta \hat{p}_{i}$. The standard uniaxial elasticity tensor, $c_{i j k l}$, has the same four terms plus a fifth one $\left(c_{5} p_{i} p_{j} p_{k} p_{l}\right)$ [28]. For the piezoelectric tensor, $\zeta_{i j k}^{E}$, we get

$$
\zeta_{i j k}^{E}=\zeta_{1}^{E}\left(\delta_{i k}^{\perp} \hat{p}_{j}+\delta_{j k}^{\perp} \hat{p}_{i}\right)+\zeta_{2}^{E} \delta_{i j}^{\perp} \hat{p}_{k}+\zeta_{3}^{E} \hat{p}_{i} \hat{p}_{j} \hat{p}_{k}
$$

with the same structure for $\zeta_{i j k}^{P}$. The direct static coupling between electric fields and relative rotations is given by

$$
\zeta_{i j}^{\Omega E}=\zeta^{\Omega E} \delta_{i j}^{\perp}
$$

where $\zeta_{i j}^{\Omega P}$ has the same structure. The second-rank susceptibility tensors $\chi_{i j}^{\rho, \sigma, c, P}$ are of the standard uniaxial form, eq. (5), while $\chi_{i j k}^{p}$ has the same structure as eq. (6). For the coupling between relative rotations and gradients of the polar preferred direction, which again does not exist in systems with a director-type preferred direction, there is

$$
\zeta_{i j k}^{\Omega p}=\zeta^{\Omega p} \delta_{i k}^{\perp} \hat{p}_{j}
$$

We note that the contribution $\sim \zeta_{i j k l}$ coupling strains and gradients of a polar preferred direction only exists in polar systems, while in systems with a non-polar preferred direction, such as in ordinary nematics, it cannot arise for parity reasons. Also the direct coupling of relative rotations to electric fields $\sim \zeta^{\Omega E}$ only exists in polar gels and not in the usual nematic elastomers. The flexoelectric term $\sim \zeta_{i j k}^{\text {flexo }}$ is of the same structure as in ordinary nematics with the director $\hat{n}_{i}$ replaced by $\hat{p}_{i}$.

The convexity of the energy requires various positivity conditions on tensor coefficients describing quadratic expressions, e.g. $\alpha>0, K_{1 \ldots 5}>0, D_{1}>0$, while those coefficients that describe bilinear cross-couplings can have either sign, but are bounded from above, e.g. $K_{6}^{2}<K_{1} K_{4}, \zeta_{1}^{2}<c_{1} K_{1},\left(\zeta_{3}^{P}\right)^{2}<c_{5} K_{4},\left(\zeta^{\Omega P}\right)^{2}<D_{1} K_{5}$, $\left(\chi_{\|}^{P} P_{0}\right)^{2}<\alpha c_{5}$, and $\left(\zeta^{\Omega p}\right)^{2}<D_{1} K_{3}$.

Equation (3) contains the energy density of a normal fluid binary mixture $\left(\varepsilon_{0}\right)$ and the polar analogues of that of a usual nematic phase including spatial modulations of the order parameter modulus: the Frank orientational elastic energy ( $\sim K_{i j k l}$ with splay, bend and twist [44]), the energy associated with gradients of the modulus $\left(\sim L_{i j}\right)[21]$ and a cross-coupling term between gradients of the preferred direction to gradients of the order parameter modulus $\left(\sim M_{i j k}\right)$ [45]. The orientation energy due to an external field is governed by $\boldsymbol{P}_{0} \cdot \boldsymbol{E}$ and the stiffness of order parameter variations is given by $\alpha$. Although the energy density expression is given in harmonic approximation only, it can give rise to nonlinear effects, since all material parameters are still functions of the state variables, like temperature, pressure, and the polarization $P_{0}$. This is in contrast to ordinary nematics, where the material parameters can only be a function of $E^{2}$.

The third to fifth line of eq. (3) contains contributions that are specific for polar nematics and are absent for ordinary, non-polar nematics as they would violate the $\hat{\boldsymbol{n}} \rightarrow-\hat{\boldsymbol{n}}$ invariance. These comprise couplings $\left(\sim \gamma_{i}\right)$ between the polarization and variations of $\rho, \sigma$ and $c$, which are of the same nature as the pyroelectric term in solids [46]. They arise from appropriate terms $\sim P^{2}$ in the Ginzburg-Landau free energy, eq. (A.1), explaining the explicit $P_{0}$ factors. Other cross-coupling terms, $\sim \bar{\beta}_{1,2,3}$ and $\sim \beta_{1,2,3}$, are relating variations of $\rho, \sigma$ and $c$ to splay and to spatial variations of the polarization along the preferred direction, $\hat{p}_{i} \nabla_{i} P$, respectively. 
For the ground state considered here, the possible linear splay contribution, $\nabla_{i} P_{i}$ to the energy is simply a surface term, which we omit. For the importance of this term in finding the ground state, cf. appendix A.

Line six contains linear elasticity, the characteristic self-coupling of relative rotations and the coupling of strains to relative rotations. In line seven we have static cross-coupling terms between strains and variations of density, entropy density, concentration and the magnitude of the polarization. These terms listed in lines six and seven are analogues and isomorphic to the case of nematic gels, when the director $\hat{n}_{i}$ is replaced by the polar preferred direction $\hat{p}_{i}$. Line eight contains three coupling terms of strains to gradients of the polar direction and gradients of $P$ as well as to external electric fields: these two cross-couplings only exist for gels with a polar preferred direction. For completeness we have also listed in line eight the analogue of the flexoelectric contribution in a nematic for a system with a polar preferred direction. In line nine we have three coupling terms of relative rotations to external electric fields and to gradients of the polar preferred direction and of $P$. All three terms only exist for polar gels with relative rotations and have not been considered before.

Ferroelectric solids are known to show electrostriction, a coupling between the polarization and elastic deformations, which can be written (in close analogy to eq. (A.2) for the magnetic case studied in ref. [28])

$$
\varepsilon_{P}=\frac{1}{2} \gamma_{i j k l}^{P} P_{i} P_{j} u_{k l}
$$

where $\gamma_{i j k l}^{P}$ has six independent coefficients $[46,47]$ in a uniaxial material. For the given ground state, this expression contains a term, which is linear in the deviations, $\sim P_{0}^{2} \hat{p}_{i} \hat{p}_{j} u_{i j}^{e s}$ that leads to an elastic, electrostrictive deformation $u_{i j}^{e s}$. We assume that in our ground state the electrostrictive deformation has already been taken into account and the strain tensor $u_{i j}$ describes deviations from this true ground state. Then, the linear contribution of eq. (11) vanishes. The contribution quadratic in deviations from the ground state, $\sim P_{0}\left(\hat{p}_{i} \delta P_{j}+\hat{p}_{j} \delta P_{i}\right) u_{i j}$, leads to the contribution $\chi_{i j}^{P} P_{0} u_{i j} \delta P$ in the energy, eq. (3), explaining also the explicit $P_{0}$ factor. We will discard the other quadratic term $\sim\left(\hat{p}_{i} \delta \hat{p}_{j}+\hat{p}_{j} \delta \hat{p}_{i}\right) u_{i j}$, leading to $\chi_{i j k}^{p} u_{i j} \delta \hat{p}_{k}$ in eq. (3), since we neglect orientation of the polarization due to elastic deformations (cf. appendix A) compared to orientation due to the external field.

The thermodynamic forces and thus simultaneously the static properties of polar nematic gels and elastomers are obtained by expanding first the generalized energy density into the macroscopic variables and then, in a second step, by taking the variational derivatives with respect to one variable while keeping all other variables fixed, denoted by ellipses in the following [21]. We get in detail in harmonic approximation

$$
v_{i}=\left.\frac{\partial \varepsilon}{\partial g_{i}}\right|_{\ldots}=\frac{1}{\rho} g_{i},
$$

$$
\begin{aligned}
& h^{\prime P}=\left.\frac{\partial \varepsilon}{\partial \delta P}\right|_{\ldots}=\alpha \delta P+\left(\gamma_{1} \delta \rho+\gamma_{2} \delta \sigma+\gamma_{3} \delta c\right) P_{0} \\
& +\chi_{i j}^{P} P_{0} u_{i j} \\
& \Phi_{i}^{P}=\left.\frac{\partial \varepsilon}{\partial\left(\nabla_{i} P\right)}\right|_{\ldots}=L_{i j} \nabla_{j} P+M_{i j k} \nabla_{j} \hat{p}_{k} \\
& +\hat{p}_{i}\left(\beta_{1} \delta \rho+\beta_{2} \delta \sigma+\beta_{3} \delta c\right) \\
& +\zeta_{i j}^{\Omega P} \tilde{\Omega}_{j}+\zeta_{k j i}^{P} u_{j k}, \\
& h_{i}^{\prime}=\left.\frac{\partial \varepsilon}{\partial \delta \hat{p}_{i}}\right|_{\ldots}=P_{0} E \delta \hat{p}_{i}, \\
& \Phi_{i j}=\left.\frac{\partial \varepsilon}{\partial\left(\nabla_{j} \hat{p}_{i}\right)}\right|_{\ldots}=K_{j i k l} \nabla_{k} \hat{p}_{l}+M_{k j i} \nabla_{k} P \\
& +\zeta_{k l j i} u_{k l}+\zeta_{j i k}^{\mathrm{flexo}} E_{k}+\zeta_{k j i}^{\Omega p} \tilde{\Omega}_{k} \\
& +\delta_{i j}^{\perp}\left(\bar{\beta}_{1} \delta \rho+\bar{\beta}_{2} \delta \sigma+\bar{\beta}_{3} \delta c\right), \\
& \delta \mu=\left.\frac{\partial \varepsilon}{\partial \delta \rho}\right|_{\ldots}=\gamma_{1} P_{0} \delta P+\beta_{1} \hat{p}_{i} \nabla_{i} P+\bar{\beta}_{1} \nabla_{i} \hat{p}_{i} \\
& +\chi_{i j}^{\rho} u_{i j} \\
& \delta T=\left.\frac{\partial \varepsilon}{\partial \delta \sigma}\right|_{\ldots}=\gamma_{2} P_{0} \delta P+\beta_{2} \hat{p}_{i} \nabla_{i} P+\bar{\beta}_{2} \nabla_{i} \hat{p}_{i} \\
& +\chi_{i j}^{\sigma} u_{i j}
\end{aligned}
$$

$$
\begin{aligned}
\delta \mu_{c}=\left.\frac{\partial \varepsilon}{\partial \delta c}\right|_{\ldots=}= & \gamma_{3} P_{0} \delta P+\beta_{3} \hat{p}_{i} \nabla_{i} P+\bar{\beta}_{3} \nabla_{i} \hat{p}_{i} \\
& +\chi_{i j}^{c} u_{i j},
\end{aligned}
$$

$$
\begin{aligned}
\psi_{i j}=\left.\frac{\partial \varepsilon}{\partial u_{i j}}\right|_{\ldots}= & c_{i j k l} u_{k l}+\chi_{i j}^{\rho} \delta \rho+\chi_{i j}^{\sigma} \delta \sigma+\chi_{i j}^{P} P_{0} \delta P \\
& +\chi_{i j}^{c} \delta c+\frac{1}{2} D_{2}\left(\delta_{i k}^{\perp} \hat{p}_{j}+\delta_{j k}^{\perp} \hat{p}_{i}\right) \tilde{\Omega}_{k} \\
& +\zeta_{i j k l} \nabla_{k} \hat{p}_{l}+\zeta_{i j k}^{E} E_{k}+\zeta_{i j k}^{P} \nabla_{k} P,
\end{aligned}
$$

$$
\begin{aligned}
L_{i}^{\perp}=\left.\frac{\partial \varepsilon}{\partial \tilde{\Omega}_{i}}\right|_{\ldots}= & D_{1} \tilde{\Omega}_{i}+\frac{1}{2} D_{2}\left(\hat{p}_{j} \delta_{i k}^{\perp}+\hat{p}_{k} \delta_{i j}^{\perp}\right) u_{j k} \\
& +\zeta_{i j}^{\Omega E} E_{j}+\zeta_{i j k}^{\Omega p} \nabla_{j} \hat{p}_{k}+\zeta_{i j}^{\Omega P} \nabla_{j} P
\end{aligned}
$$

from which the total molecular fields $W=h^{\prime P}-\nabla_{j} \Phi_{j}^{P}$ and $h_{i}^{p}=h_{i}^{\prime}-\nabla_{j} \Phi_{i j}$ follow immediately.

\section{Dynamics}

\subsection{Dynamic equations}

The macroscopic equations for conserved quantities, variables associated with spontaneously broken continuous 
symmetries and slowly relaxing variables are

$$
\begin{aligned}
\frac{\partial}{\partial t} \rho+\operatorname{div} \rho \boldsymbol{v} & =0, \\
\left(\frac{\partial}{\partial t}+v_{i} \nabla_{i}\right) \rho^{\mathrm{el}}+\operatorname{div} \boldsymbol{j}^{\mathrm{el}} & =0, \\
\frac{\partial}{\partial t} \sigma+\operatorname{div} \sigma \boldsymbol{v}+\operatorname{div} \boldsymbol{j}^{\sigma} & =\frac{2 R}{T}, \\
\frac{\partial}{\partial t} g_{i}+\nabla_{j}\left(v_{j} g_{i}+\delta_{i j} \Pi-\psi_{i j}+\sigma_{i j}^{\mathrm{th}}+\sigma_{i j}\right) & =0, \\
\left(\frac{\partial}{\partial t}+v_{j} \nabla_{j}\right) \hat{p}_{i}+(\hat{\boldsymbol{p}} \times \boldsymbol{\omega})_{i}+X_{i} & =0, \\
\rho\left(\frac{\partial}{\partial t}+v_{j} \nabla_{j}\right) c+\operatorname{div} \boldsymbol{j}^{c} & =0, \\
\left(\frac{\partial}{\partial t}+v_{j} \nabla_{j}\right) P+X^{P} & =0, \\
\left(\frac{\partial}{\partial t}+v_{j} \nabla_{j}\right) \tilde{\Omega}_{i}+Y_{i}^{\Omega} & =0, \\
\left(\frac{\partial}{\partial t}+v_{j} \nabla_{j}\right) u_{i j}-A_{i j}+X_{i j}^{u} & =0,
\end{aligned}
$$

with $g_{i}=\rho v_{i}$ and $[28,35]$

$$
\begin{aligned}
\sigma_{i j}^{\mathrm{th}}= & -E_{j} D_{i}+\Phi_{k j} \nabla_{i} p_{k}+2 \psi_{j k} u_{k i} \\
& -\frac{1}{2}\left(\hat{p}_{j} h_{i}^{p}-\hat{p}_{i} h_{j}^{p}\right)-\frac{1}{2}\left(\tilde{\Omega}_{j} L_{i}^{\perp}-\tilde{\Omega}_{i} L_{j}^{\perp}\right),
\end{aligned}
$$

where $A_{i j}=\frac{1}{2}\left(\nabla_{i} v_{j}+\nabla_{j} v_{i}\right)$ is the deformational flow and $\omega_{i}=\frac{1}{2} \epsilon_{i j k} \nabla_{j} v_{k}$ the vorticity. The thermodynamic pressure is defined as $\Pi \equiv-(\partial / \partial V) \int \varepsilon \mathrm{d} V$ and given by

$$
\Pi=-\varepsilon+T \sigma+\mu \rho+\mu_{c} c+\boldsymbol{g} \cdot \boldsymbol{v}+\boldsymbol{E} \cdot \boldsymbol{D} .
$$

It only contains the extensive degrees of freedom. Note that the thermodynamic pressure is not equal to (one third of) the trace of the total stress tensor, due to the elastic stress $\psi_{i j}$, eq. (20), and the nonlinearities in eq. (31). The parts of the currents shown explicitly in (22)-(31) are not material dependent, but are given by general symmetry and thermodynamic principles [21], like transformation behavior under translations (transport terms) or rotations (convective terms) and by the requirement of zero entropy production $(R=0)$ of all those terms together with the isotropic pressure term in eq. (25).

Using the requirement [21]

$$
\begin{aligned}
& \omega_{i j}\left(-P_{i} E_{j}+h_{i}^{p} \hat{p}_{j}+\Phi_{k i} \nabla_{j} \hat{p}_{k}\right. \\
& \left.\quad+\nabla_{k}\left(\hat{p}_{j} \Phi_{i k}\right)+L_{i}^{\perp} \tilde{\Omega}_{j}+2 \psi_{k i} u_{k j}\right)=0
\end{aligned}
$$

for any constant antisymmetric matrix $\omega_{i j}=-\omega_{j i}$, which ensures the rotational invariance of the Gibbs relation, eq. (1), the non-symmetric part of the stress tensor, eq. (31), can be transformed as

$$
\begin{aligned}
2 \sigma_{i j}^{\mathrm{th}}= & -\left(E_{j} D_{i}+E_{i} D_{j}\right)+\Phi_{k i} \nabla_{j} \hat{p}_{k}+\Phi_{k j} \nabla_{i} \hat{p}_{k} \\
& +2\left(\psi_{j k} u_{k i}+\psi_{i k} u_{k j}\right)+\nabla_{k}\left(\hat{p}_{j} \Phi_{i k}-\hat{p}_{i} \Phi_{j k}\right) .
\end{aligned}
$$

Now $\sigma_{i j}^{\text {th }}$ is either symmetric or a divergence of an antisymmetric part, which ensures angular momentum conservation. It can be brought into a manifestly symmetric form by some redefinitions [19].

The source term $2 R / T$ in (24) is the entropy production, which has to be zero for reversible, and positive for irreversible processes. The phenomenological parts of the entropy current $j_{i}^{\sigma}$, the stress tensor $\sigma_{i j}$, the concentration current $j_{i}^{c}$ and the quasi-currents $X_{i}, Y_{i}^{\Omega}, X^{P}$, and $X_{i j}^{u}$, associated with the temporal changes of the polar unit vector, relative rotations, the polar nematic order, and the strain tensor, respectively, are given below. These phenomenological currents and quasi-currents can be split into reversible (superscript $R$ ) and dissipative parts (superscript $D$ ), where the former have the same time reversal behavior as the time derivative of the appropriate variable and must give $R=0$, while the latter have the opposite behavior and give $R>0$. The phenomenological currents and quasi-currents are given within "linear irreversible thermodynamics" (guaranteeing general Onsager relations), i.e. as linear relations between currents and thermodynamic forces. The resulting expressions are nevertheless nonlinear, since all material parameters can be functions of the state variables $(e . g . \Pi, T, P)$. The phenomenological part of the stress tensor $\sigma_{i j}$ has to be symmetric guaranteeing angular momentum conservation. The dynamic equation for the energy density follows from eqs. (22)-(30) via eq. (1), and is not shown here.

\subsection{Reversible dynamics}

Making use of symmetry arguments (including behavior under time reversal, parity, rigid rotations, rigid translations and covariance under Galilei transformations) and Onsager's relations we obtain the following expressions for the reversible currents up to linear order in the thermodynamic forces

$$
\begin{aligned}
g_{i}= & \rho v_{i}, \\
j_{i}^{\sigma R}= & \varphi_{i j k}^{\sigma} A_{j k}, \\
j_{i}^{c R}= & \varphi_{i j k}^{c} A_{j k}, \\
j_{i}^{\mathrm{el} R}= & \varphi_{i j k}^{\mathrm{el}} A_{j k}, \\
X_{i j}^{u R}= & 0, \\
\sigma_{i j}^{R}= & -\frac{1}{2} \lambda_{k j i}^{p} h_{k}^{p}+\beta_{i j} W-\frac{1}{2} \lambda^{\perp}\left(L_{i}^{\perp} \hat{p}_{j}+L_{j}^{\perp} \hat{p}_{i}\right) \\
& -\varphi_{k j i}^{\sigma} \nabla_{k} T-\varphi_{k j i}^{c} \nabla_{k} \mu_{c}-\varphi_{k j i}^{\mathrm{el}} E_{k}, \\
X^{P R}= & \beta_{i j} A_{i j}, \\
Y_{i}^{R}= & -\frac{1}{2} \lambda_{i j k}^{p} A_{j k}, \\
Y_{i}^{\Omega R}= & -\frac{1}{2} \lambda^{\perp}\left(\delta_{i j}^{\perp} \hat{p}_{k}+\delta_{i k}^{\perp} \hat{p}_{j}\right) A_{j k},
\end{aligned}
$$

with $A_{j k}=\frac{1}{2}\left(\nabla_{i} v_{k}+\nabla_{k} v_{i}\right)$. The coupling of the polarization and the density of linear momentum is provided by the tensors 


$$
\lambda_{i j k}=\lambda\left(\hat{p}_{j} \delta_{i k}^{\perp}+\hat{p}_{k} \delta_{i j}^{\perp}\right) \quad \text { and } \quad \lambda_{i j}^{P}=\lambda_{2}^{P} \delta_{i j}^{\perp}+\lambda_{3}^{P} \hat{p}_{i} \hat{p}_{j} .
$$

One finds a total of three material-dependent coupling terms. The first is the analogue of the classical flow alignment term coupling the orientation of the preferred direction to deformational flow, while the coupling to rotational flow (rigid rotation) is not material dependent and has already been made explicit in eq. (26). The two contributions $\sim \lambda_{2}^{P}$ and $\sim \lambda_{3}^{P}$ are associated with the coupling of the magnitude of the polarization, $P$, to velocity gradients (compare also the detailed discussion in the next section). We note that this coupling between the density of linear momentum and the polarization is identical in structure to that of a uniaxial nematic, when formally $\hat{p}_{i}$ is replaced by the director $\hat{n}_{i}$ and $P$ by the nematic order parameter modulus $S$. The coupling of relative rotations to flow, provided by the $\lambda^{\perp}$ terms, has already been given in nematic elastomers [28].

The tensors $\varphi_{i j k}^{\alpha}$ have been shown before to arise for polar nematics [48] and are of the structure

$$
\varphi_{i j k}^{\alpha}=\varphi_{1}^{\alpha} \hat{p}_{i} \hat{p}_{j} \hat{p}_{k}+\varphi_{2}^{\alpha} \hat{p}_{i} \delta_{j k}^{\perp}+\varphi_{3}^{\alpha}\left(\hat{p}_{j} \delta_{i k}^{\perp}+\hat{p}_{k} \delta_{i j}^{\perp}\right) .
$$

These reversible dynamic cross-coupling terms exist in all macroscopic systems with a parity breaking vector.

The physical meaning of some of these reversible couplings will be explored in sect. 4 .

\subsection{Irreversible dynamics and entropy production}

For the derivation of the dissipative parts of the phenomenological currents one usually expands the dissipation function $R$ to second order in the thermodynamic forces and then obtains the dissipative currents by taking the variational derivatives with respect to the forces. We find for the dissipation function

$$
\begin{aligned}
R= & \frac{1}{2} \kappa_{i j}\left(\nabla_{i} T\right)\left(\nabla_{j} T\right)+\frac{1}{2} \nu_{i j k l} A_{i j} A_{k l}+\frac{1}{2} \kappa_{W} W^{2} \\
& +\frac{1}{2} \gamma_{i j}\left(\nabla_{k} \psi_{i k}\right)\left(\nabla_{l} \psi_{j l}\right)+D_{i j}^{T}\left(\nabla_{j} T\right)\left(\nabla_{i} \mu_{c}\right) \\
& +\lambda_{i j}^{W T}\left(\nabla_{j} W\right)\left(\nabla_{i} T\right)+\lambda_{i j}^{W \mu}\left(\nabla_{j} W\right)\left(\nabla_{i} \mu_{c}\right) \\
& +\left(\nabla_{j} \psi_{i j}\right)\left(\xi_{i k}^{T} \nabla_{k} T+\xi_{i k}^{c} \nabla_{k} \mu_{c}+\xi_{i k}^{W} \nabla_{k} W+\xi_{i k}^{E} E_{k}\right) \\
& +\frac{1}{2} D_{i j}\left(\nabla_{i} \mu_{c}\right)\left(\nabla_{j} \mu_{c}\right)+\frac{1}{2} s_{i j} E_{i} E_{j}+D_{i j}^{E} E_{i} \nabla_{j} \mu_{c} \\
& +D_{i j}^{W} E_{i} \nabla_{j} W+\kappa_{i j}^{E} E_{i} \nabla_{j} T+\xi_{i j}^{E} E_{i} \nabla_{k} \psi_{j k} \\
& +\kappa_{\|}^{E W} \hat{p}_{i} E_{i} W+\kappa_{\|}^{P} \hat{p}_{i} W \nabla_{i} T \\
& +D_{\|}^{\psi W} \hat{p}_{i} W \nabla_{j} \psi_{i j}+D_{\|}^{P} \hat{p}_{i} W \nabla_{i} \mu_{c} \\
& +h_{k}^{p} \delta_{j k}^{\perp}\left(\xi^{p \psi} \nabla_{i} \psi_{i j}+\xi^{p T} \nabla_{j} T+\xi^{p E} E_{j}\right. \\
& +L_{k}^{\perp} \delta_{j k}^{\perp}\left(\xi^{\Omega \psi} \nabla_{i} \mu+\xi^{p W} \nabla_{j} W\right) \\
& +\xi^{\Omega T} \nabla_{j} T+\xi^{\Omega E} E_{j} \\
& +\delta_{i j}^{\perp}\left(\frac{1}{2 \gamma_{1}} h_{i}^{p} h_{j}^{p}+\frac{1}{2} \xi^{\perp} L_{i}^{\perp} L_{j}^{\perp}+\xi_{12} L_{i}^{\perp} h_{j}^{p}\right) .
\end{aligned}
$$

Here $\nu_{i j k l}$ is the uniaxial viscosity tensor [19], $\kappa_{W}$ describes the relaxation of the polar order parameter, $\kappa_{i j}$, $\gamma_{i j}, D_{i j}, D_{i j}^{T}, s_{i j}$ and $\kappa_{i j}^{E}$ describe heat conduction, strain diffusion, mass diffusion, thermodiffusion, electric conductivity and the Peltier effect, respectively. $\lambda_{i j}^{W T}, \lambda_{i j}^{W \mu}$, $\xi_{i j}^{T}, \xi_{i j}^{c}, \xi_{i j}^{W}, D_{i j}^{E}$ and $D_{i j}^{W}$, describe diffusive coupling terms between gradients of the polar order parameter and defect diffusion on one hand, and gradients of temperature and chemical potential or electric fields on the other. All second-rank dissipative property tensors discussed so far are of the uniaxial form $b_{i j}^{D}=b_{\perp}^{D} \delta_{i j}^{\perp}+$ $b_{\|}^{D} \hat{p}_{i} \hat{p}_{j} \cdot \gamma_{1}$ is associated with the diffusion or relaxation of the polar direction, $\xi^{\perp}$ characterizes the relaxation of relative rotations and the contribution $\sim \xi_{12}$ represents the coupling between relative rotations and diffusion/relaxation of the polar direction. The last three contributions are isomorphic in structure to the case of nematic elastomers with the director $\hat{n}_{i}$ replaced by $\hat{p}_{i}$ [28]. The contributions $\sim \kappa_{\|}^{E W}, \kappa_{\|}^{P}, D_{\|}^{\psi W}$ and $D_{\|}^{P}$ couple variations of relaxing polar order parameter to electric field, temperature gradients, gradients of the chemical potential and strain diffusion. The terms containing $\kappa_{\|}^{P}$ and $D_{\|}^{P}$ have been given before for polar nematic in ref. [27].

The positivity requirement for the dissipation function requires various positivity conditions on tensor coefficients describing quadratic expressions, e.g. $\kappa_{\perp, \|}, \gamma_{\perp, \|}$, $s_{\perp, \|}$, and $\kappa_{W}$ are all positive, while those coefficients that describe bilinear cross-couplings can have either sign, but are bounded from above, e.g. $\left(\kappa_{\|}^{E W}\right)^{2}<s_{\|} \kappa_{W},\left(\kappa_{\|}^{P}\right)^{2}<$ $\kappa_{\|} \kappa_{W}$, and $\left(D_{\|}^{\psi W}\right)^{2}<\gamma_{\|} \kappa_{W}$.

All terms in eq. (46) $\sim \xi^{\Omega x} L_{k}^{\perp}$ coupling relative rotations to temperature gradients, electric fields, gradients of chemical potential and polar order parameter as well as to strain diffusion are given here for the first time. They are characteristic for systems with polar order and relative rotations. The contributions $\sim \xi^{p \alpha} h_{k}^{p}$ represent cross couplings between the molecular field of the polar direction $\left(h_{k}^{p}\right)$ and temperature gradients, electric fields, gradients of chemical potential and polar order parameter as well as strain diffusion. Two of these contributions $(\sim \nabla T$ and $\nabla \mu)$ have been presented for polar nematic [27]. All terms associated with $\xi^{p x}$ are characteristic for systems with a polar direction and do not arise for non-polar liquid crystals.

Inspecting carefully eq. (46) one notes that, except for the dissipation associated with the extensional flow, $A_{i j}$, all macroscopic variables couple to each other dissipatively to a low order in the wave vector.

The range of possible values of the coefficients in eq. (46) is restricted by the positivity of the entropy production.

To obtain the dissipative parts of the currents and quasi-currents we take the partial derivatives of $R$ with 
respect to the appropriate thermodynamic force

$$
\begin{aligned}
& j_{i}^{\sigma D}=-\kappa_{i j} \nabla_{j} T-D_{i j}^{T} \nabla_{j} \mu_{c}-\kappa_{i j}^{E} E_{j} \\
& -\lambda_{i j}^{W T} \nabla_{j} W-\xi_{i j}^{T} \nabla_{k} \psi_{j k} \\
& -\xi^{p T} \delta_{i j}^{\perp} h_{j}^{p}-\xi^{\Omega T} \delta_{i j}^{\perp} L_{j}^{\perp}-\kappa_{\|}^{p} \hat{p}_{i} W, \\
& j_{i}^{c D}=-D_{i j} \nabla_{j} \mu_{c}-D_{i j}^{T} \nabla_{j} T-D_{i j}^{E} E_{j} \\
& -\lambda_{i j}^{W \mu} \nabla_{j} W-\xi_{i j}^{c} \nabla_{k} \psi_{j k} \\
& -\xi^{p \mu} \delta_{i j}^{\perp} h_{j}^{p}-\xi^{\Omega \mu} \delta_{i j}^{\perp} L_{j}^{\perp}-D_{\|}^{p} \hat{p}_{i} W, \\
& j_{i}^{\mathrm{el} D}=\sigma_{i j} E_{j}+D_{i j}^{E} \nabla_{j} \mu_{c}+\kappa_{i j}^{E} \nabla_{j} T \\
& +D_{i j}^{W} \nabla_{j} W+\xi_{i j}^{E} \nabla_{k} \psi_{j k} \\
& +\xi^{p E} \delta_{i j}^{\perp} h_{j}^{p}+\xi^{\Omega E} \delta_{i j}^{\perp} L_{j}^{\perp}+\kappa_{\|}^{E W} \hat{p}_{i} W, \\
& \sigma_{i j}^{D}=-\nu_{i j k l}^{D} A_{k l}, \\
& Y_{i}^{D}=\frac{1}{\gamma_{1}} \delta_{i j}^{\perp} h_{j}^{p}+\xi_{12} L_{i}^{\perp} \\
& +\xi^{p \psi} \delta_{i j}^{\perp} \nabla_{k} \psi_{k j}+\xi^{p T} \delta_{i j}^{\perp} \nabla_{j} T+\xi^{p E} \delta_{i j}^{\perp} E_{j} \\
& +\xi^{p \mu} \delta_{i j}^{\perp} \nabla_{j} \mu+\xi^{p W} \delta_{i j}^{\perp} \nabla_{j} W, \\
& Y_{i}^{\Omega D}=\xi^{\perp} L_{i}^{\perp}+\xi_{12} h_{i}^{n} \\
& +\xi^{\Omega \psi} \delta_{i j}^{\perp}\left(\nabla_{k} \psi_{k j}\right)+\xi^{\Omega T} \delta_{i j}^{\perp}\left(\nabla_{j} T\right)+\xi^{\Omega E} \delta_{i j}^{\perp} E_{j} \\
& +\xi^{\Omega \mu} \delta_{i j}^{\perp}\left(\nabla_{j} \mu\right)+\xi^{\Omega W} \delta_{i j}^{\perp}\left(\nabla_{j} W\right), \\
& Z^{D}=\kappa_{W} W-\nabla_{j}\left(\lambda_{i j}^{W T} \nabla_{i} T+\lambda_{i j}^{W \mu} \nabla_{i} \mu_{c}\right) \\
& -\nabla_{k}\left(\xi_{i k}^{W} \nabla_{j} \psi_{i j}\right)-\nabla_{j}\left(D_{i j}^{W T} E_{i}\right) \\
& +\kappa_{\|}^{E W} \hat{p}_{i} E_{i}+\kappa_{\|}^{P} \hat{p}_{i} \nabla_{i} T \\
& +D_{\|}^{\psi W} \hat{p}_{i} \nabla_{j} \psi_{i j}+D_{\|}^{P} \hat{p}_{i} \nabla_{i} \mu_{c} \\
& -\nabla_{j}\left(\xi^{p W} \delta_{j k}^{\perp} h_{k}^{p}\right)-\nabla_{j}\left(\xi^{\Omega W} \delta_{j k}^{\perp} L_{k}^{\perp}\right), \\
& X_{i j}^{u D}=-\frac{1}{2}\left[\nabla _ { j } \left(\xi_{i k}^{T} \nabla_{k} T+\xi_{i k}^{c} \nabla_{k} \mu_{c}+\xi_{i k}^{W} \nabla_{k} W\right.\right. \\
& +\xi_{i k}^{E} E_{k}+\gamma_{i k} \nabla_{l} \psi_{k l}+D_{\|}^{\psi W} \hat{p}_{i} W \\
& \left.\left.+\xi^{p \psi} \delta_{i k}^{\perp} h_{k}^{p}+\xi^{\Omega \psi} \delta_{i k}^{\perp} L_{k}^{\perp}\right)+(i \leftrightarrow j)\right] .
\end{aligned}
$$

\section{Experimental considerations}

As a first important observation we notice that there is only one macroscopic variable, which is odd under time reversal, namely the density of momentum $g_{i}$ or, equivalently, the velocity $v_{i}$. In addition, there is no intrinsic unit vector in the system, which is odd under time reversal and which would facilitate reversible cross-coupling terms. Examples for such systems include ferromagnets and antiferromagnets [20], the superfluid phases ${ }^{3} \mathrm{He}-\mathrm{A}$ and ${ }^{3} \mathrm{He}-\mathrm{A}_{1}[24,49]$ and uniaxial magnetic gels [50].

\subsection{Reversible cross-coupling terms}

First we discuss briefly the question of reversible crosscoupling terms. It turns out that all reversible crosscoupling arising and listed in eqs. (36)-(38) and (40)-(43) have been presented before for nematic gels [28] or for low molecular weight polar nematic liquid crystals $[27,48]$. We refer to these references for a more detailed analysis.

\subsection{Physical consequences of selected static cross-coupling terms}

Inspecting the various static cross-coupling terms in eq. (3), we find that there are four types which are characteristic for gels with polar preferred directions and which do not exist in nematic gels or low molecular weight polar nematics. One of them, namely piezoelectricity, the static coupling between the strain tensor and electric fields, is of the same structure as the piezoelectric tensor in solid state physics. It thus has for a uniaxial polar system of the nature discussed here three independent piezoelectric coefficients [46].

There is one novel static coupling to strains, namely the coupling between spatial variations of the polar preferred direction and strains. The corresponding terms take the form

$$
\begin{aligned}
& \Phi_{i j}=\ldots-\zeta_{k l j i} u_{i j}, \\
& \psi_{i j}=\ldots+\zeta_{i j k l} \nabla_{k} \hat{p}_{l},
\end{aligned}
$$

where $\zeta_{i j k l}$ has four independent coefficients (compare eq. (7)).

Relative rotations are associated with two static crosscoupling terms not considered before. They can couple to external electric fields as well as to spatial variations of the polar preferred direction. Specifically we obtain

$$
\begin{aligned}
& L_{i}^{\perp}=\ldots+\zeta_{i j}^{\Omega E} E_{j}+\zeta_{i j k}^{\Omega p} \nabla_{j} \hat{p}_{k}, \\
& \Phi_{i j}=\ldots+\zeta_{k j i}^{\Omega p} \tilde{\Omega}_{k},
\end{aligned}
$$

where

$$
\zeta_{i j}^{\Omega E}=\zeta^{\Omega E} \delta_{i j}^{\perp}
$$

and

$$
\zeta_{i j k}^{\Omega p}=\zeta^{\Omega p} \delta_{i k}^{\perp} \hat{p}_{j}
$$

To get a better intuition for the experimental consequences of these cross-coupling terms we consider a concrete geometry. We take the equilibrium value of the polar preferred direction to be parallel to the $\hat{z}$-axis. Then we have

$$
\begin{aligned}
L_{x}^{\perp} & =\ldots+\zeta^{\Omega E} E_{x}+\zeta^{\Omega p} \nabla_{z} \hat{p}_{x} \\
L_{y}^{\perp} & =\ldots+\zeta^{\Omega E} E_{y}+\zeta^{\Omega p} \nabla_{z} \hat{p}_{y} \\
\Phi_{x z} & =\ldots+\zeta^{\Omega p} \tilde{\Omega}_{x} \\
\Phi_{y z} & =\ldots+\zeta^{\Omega p} \tilde{\Omega}_{y} .
\end{aligned}
$$


From eqs. (61) to (64) we arrive at the following three conclusions for the novel static behavior for polar gels. An electric field applied perpendicularly to the polar preferred direction leads to relative rotations. Relative rotations lead to bend deformations of the polar direction and, conversely, bend deformations of the director lead to relative rotations. None of these effects is possible for the usual nematic gels.

\subsection{Physical consequences of selected dissipative cross-coupling terms}

For the dissipative cross-coupling terms between relative rotations and the molecular field associated with the polar direction on one hand and gradients of temperature, relative chemical potential, elastic stress and order parameter field and electric fields we have as the relevant subset of eqs. (47) to (49) and (51) to (54)

$$
\begin{aligned}
j_{i}^{\mathrm{el} D}= & -\xi_{i j}^{p E} h_{j}^{p}-\xi_{i j}^{\Omega E} L_{j}^{\perp}, \\
j_{i}^{\sigma D}= & -\xi_{i j}^{p T} h_{j}^{p}-\xi_{i j}^{\Omega T} L_{j}^{\perp}, \\
j_{i}^{c D}= & -\xi_{i j}^{p \mu} h_{j}^{p}-\xi_{i j}^{\Omega \mu} L_{j}^{\perp}, \\
Y_{i}^{D}= & +\xi_{i j}^{p \psi} \nabla_{k} \psi_{k j}+\xi_{i j}^{p T} \nabla_{j} T+\xi_{i j}^{p E} E_{j} \\
& +\xi_{i j}^{p \mu} \nabla_{j} \mu+\xi_{i j}^{p W} \nabla_{j} W, \\
Y_{i}^{\Omega D}= & +\xi_{i j}^{\Omega \psi} \nabla_{k} \psi_{k j}+\xi_{i j}^{\Omega T} \nabla_{j} T+\xi_{i j}^{\Omega E} E_{j} \\
& +\xi_{i j}^{\Omega \mu} \nabla_{j} \mu+\xi_{i j}^{\Omega W} \nabla_{j} W, \\
Z^{D}= & -\nabla_{j}\left(\xi_{j k}^{p W} h_{k}^{p}\right)-\nabla_{j}\left(\xi_{j k}^{\Omega W} L_{k}^{\perp}\right), \\
X_{i j}^{u D}= & \frac{1}{2}\left[\left(\xi_{i k}^{p \psi} h_{k}^{p}+\xi_{i k}^{\Omega \psi} L_{k}^{\perp}\right)+(i \leftrightarrow j)\right] .
\end{aligned}
$$

Inspecting eqs. (65) to (71) we see that, for example, heat currents and electric currents arise due to relative rotations and, perhaps easier to check experimentally, temperature gradients and electric fields lead to relative rotations. Similarly deformations of the polar direction lead to heat, concentration and electric currents.

To arrive at a simple picture for the experimental consequences of these dissipative cross-coupling terms we consider a simple geometry. We again take the equilibrium value of the polar preferred direction to be parallel to the $\hat{z}$-axis. Then we have

$$
\begin{aligned}
& j_{x}^{\mathrm{el} D}=-\xi^{p E} h_{x}^{p}-\xi^{\Omega E} L_{x}^{\perp}, \\
& j_{y}^{\mathrm{el} D}=-\xi^{p E} h_{y}^{p}-\xi^{\Omega E} L_{y}^{\perp} .
\end{aligned}
$$

That is, relative rotations and the molecular field associated with the polar direction give rise to tranverse electric currents. In addition

$$
\begin{aligned}
Y_{x}^{D} & =+\xi^{p E} E_{x}, \\
Y_{y}^{D} & =+\xi^{p E} E_{y}, \\
Y_{x}^{\Omega D} & =+\xi^{\Omega T} \nabla_{x} T+\xi^{\Omega E} E_{x}, \\
Y_{y}^{\Omega D} & =+\xi^{\Omega T} \nabla_{y} T+\xi^{\Omega E} E_{y} .
\end{aligned}
$$

Thus, electric fields and temperature gradients perpendicular to the polar direction give rise to relative rotations and finite values of the molecular field.

We close this section by emphasizing that all these effects can only arise for polar gels.

\section{Influence of a transient network on the macroscopic dynamics}

In this brief section we outline the changes for the macroscopic behavior of polar nematic gels and elastomers for the case that a transient network -in addition to a permanent network - is present.

As usual for the case of transient networks the static considerations are unchanged. The dynamic equation for the strain tensor, eq. (30), can now have a source term, since the network relaxes. To leading order in the wave vector the reversible dynamics in the presence of a transient network does not change.

For the dissipation function, however, there are several additions, which are of lower order in $k$ than for the case of gels and elastomers

$$
\begin{aligned}
R= & \ldots+\frac{1}{2}\left(\tau^{-1}\right)_{i j k l} \psi_{i j} \psi_{k l} \\
& +\psi_{i j}\left(\tau_{i j k}^{T} \nabla_{k} T+\tau_{i j k}^{c} \nabla_{k} \mu_{c}+\tau_{i j k}^{W} \nabla_{k} W+\tau_{i j k}^{E} E_{k}\right) \\
& +\psi_{i j}\left(\tau_{i j k}^{p \psi} h_{k}^{p}+\tau_{i j k}^{\Omega \psi} L_{k}^{\perp}\right)
\end{aligned}
$$

In eq. (78) the tensor associated with the relaxation times of the transient network, $\left(\tau^{-1}\right)_{i j k l}$ has the same structure as the tensor of the elastic moduli, $c_{i j k l}$. The third-rank tensors in the second line of eq. (78) take the form

$$
\tau_{i j k}^{\xi \psi}=\tau_{1}^{\xi \psi}\left(\hat{p}_{i} \delta_{j k}^{\perp}+\hat{p}_{j} \delta_{i k}^{\perp}\right)+\tau_{2}^{\xi \psi} \hat{p}_{i} \hat{p}_{j} \hat{p}_{k}
$$

while the third-rank tensors in the third line of eq. (78) take the form

$$
\tau_{i j k}^{\xi}=\tau^{\xi}\left(\hat{p}_{i} \delta_{j k}^{\perp}+\hat{p}_{j} \delta_{i k}^{\perp}\right) .
$$

Taking the derivatives of the dissipation function eq. (78) we obtain for the contributions from the transient network to the dissipative parts of the currents and quasi-currents

$$
\begin{aligned}
j_{k}^{\sigma D}= & -\tau_{i j k}^{T} \psi_{i j}, \\
j_{k}^{c D}= & -\tau_{i j k}^{c} \psi_{i j}, \\
j_{k}^{\mathrm{el} D}= & \tau_{i j k}^{E} \psi_{i j}, \\
Y_{k}^{D}= & \tau_{i j k}^{p \psi} \psi_{i j}, \\
Y_{k}^{\Omega D}= & \tau_{i j k}^{\Omega \psi} \psi_{i j}, \\
X^{P D}= & -\nabla_{k}\left(\tau_{i j k}^{W} \psi_{i j}\right), \\
X_{i j}^{u D}= & \left(\tau^{-1}\right)_{i j k l} \psi_{k l} \\
& +\tau_{i j k}^{T} \nabla_{k} T+\tau_{i j k}^{c} \nabla_{k} \mu_{c}+\tau_{i j k}^{W} \nabla_{k} W+\tau_{i j k}^{E} E_{k} \\
& +\tau_{i j k}^{p \psi} h_{k}^{p}+\tau_{i j k}^{\Omega \psi} L_{k}^{\perp} .
\end{aligned}
$$


We point out that none of the cross-coupling terms entering eqs. (81)-(87) are possible for non-polar transient nematic gels and elastomers. Specifically these contributions predict that in a polar system the mechanical stresses arising from a transient network give rise to heat and electric currents.

\section{Macroscopic dynamics of chiral polar gels}

In this section we discuss chiral contributions, which arise when, in addition to the polar preferred direction, a pseudoscalar quantity, $q_{0}$, is present in polar nematic networks. In any system with orientational order order chirality allows for a linear twist energy that can lead to a helical structure of the preferred direction (compare, for example, for systems with a director: [51]). In addition, the twist couples to all scalar variables (static Lehmann effects) $[52-55]$ and to strains and relative rotations

$$
\begin{aligned}
\varepsilon_{c h}= & q_{0} L_{2} \hat{\boldsymbol{p}} \cdot(\boldsymbol{\nabla} \times \hat{\boldsymbol{p}})+q_{0} K_{7}(\boldsymbol{\nabla} \cdot \hat{\boldsymbol{p}})[\hat{\boldsymbol{p}} \cdot(\boldsymbol{\nabla} \times \hat{\boldsymbol{p}})] \\
& -q_{0}\left(\tau_{c} \delta c+\tau_{\sigma} \delta \sigma+\tau_{\rho} \delta \rho+\tau_{P} \delta P\right) \hat{\boldsymbol{p}} \cdot(\boldsymbol{\nabla} \times \hat{\boldsymbol{p}}) \\
& -q_{0} \tau_{i j}^{u} \hat{\boldsymbol{p}} \cdot(\boldsymbol{\nabla} \times \hat{\boldsymbol{p}}) u_{i j}-q_{0} \tau_{\Omega} \epsilon_{i k m} \hat{p}_{j} \hat{p}_{m} \tilde{\Omega}_{i} \nabla_{j} \hat{p}_{k} .
\end{aligned}
$$

The pitch of the helix generally is $\sim q_{0}$ and becomes exactly $q_{0}$, if the coefficient of the linear twist term, $L_{2}$, is identical to the quadratic twist elastic coefficient $K_{2}$ [56]. The contribution $\sim K_{7}$ is unique to systems, which possess both, a polar preferred direction as well as a pseudoscalar quantity $q_{0}$. It couples splay and twist and was given without any further discussion first in the classical paper by Frank [57] and denoted there by $K_{12}$. The contribution $\sim \tau_{P}$ is associated with variations of the modulus of the order parameter and arises for all helical systems.

The contributions in the last line of eq. (88) are specific for chiral elastic systems and have been given first very recently for ferrocholesteric liquid crystals and for ferrocholesteric networks [58], which are characterized by a director $n_{i}$. The first one represents a coupling of twist to the strain tensor $u_{i j}$, where $\tau_{i j}^{u}$ takes the form

$$
\tau_{i j}^{u}=\tau_{1}^{u} \hat{p}_{i} \hat{p}_{j}+\tau_{2}^{u} \delta_{i j}^{\perp} .
$$

Thus this term gives rise to changes in the pitch due to uniaxial mechanical stresses such as compression and dilatation. Such an effect has been studied for cholesteric sidechain elastomers in detail experimentally [59,60]. The second one $\left(\sim \tau_{\Omega}\right)$ relates static director deformations with relative rotations.

Other static effects specific for general chiral elastic systems are related to electric fields

$$
\varepsilon_{D c h}=q_{0} \zeta^{\Omega} \hat{p}_{j} \epsilon_{i j k} D_{i} \tilde{\Omega}_{k}+q_{0} \zeta_{i j k}^{\psi} D_{i} u_{j k}
$$

and describe electric field-induced relative rotations (rotato-electricity $[61,62]$ ) and deformations with

$$
\zeta_{i j k}^{\psi}=\zeta^{\psi}\left(\epsilon_{i j r} \hat{p}_{r} \hat{p}_{k}+\epsilon_{i k r} \hat{p}_{r} \hat{p}_{j}\right) .
$$

Next we give the additional chiral contributions to the thermodynamic conjugate quantities that arise from the chiral energy $\varepsilon=\varepsilon_{c h}+\varepsilon_{D c h}$, eqs. (88)-(91), by taking the variational derivative with respect to the appropriate variables

$$
\begin{aligned}
\delta T= & -q_{0} \tau_{\sigma} \hat{\boldsymbol{p}} \cdot(\boldsymbol{\nabla} \times \hat{\boldsymbol{p}}), \\
\delta \mu= & -q_{0} \tau_{\rho} \hat{\boldsymbol{p}} \cdot(\boldsymbol{\nabla} \times \hat{\boldsymbol{p}}), \\
\delta \mu_{c}= & -q_{0} \tau_{c} \hat{\boldsymbol{p}} \cdot(\boldsymbol{\nabla} \times \hat{\boldsymbol{p}}), \\
W= & -q_{0} \tau_{P} \hat{\boldsymbol{p}} \cdot(\boldsymbol{\nabla} \times \hat{\boldsymbol{p}}), \\
\psi_{i j}= & -q_{0} \tau_{i j}^{u} \hat{\boldsymbol{p}} \cdot(\boldsymbol{\nabla} \times \hat{\boldsymbol{p}})+q_{0} \zeta_{k i j}^{\psi} D_{k}, \\
L_{i}^{\perp}= & +q_{0} \epsilon_{j k i}\left(\zeta^{\Omega} D_{j}+\tau_{\Omega} \hat{p}_{j} \hat{p}_{r} \nabla_{r}\right) \hat{p}_{k}, \\
E_{i}= & q_{0} \zeta^{\Omega} \hat{p}_{j} \epsilon_{i j k} \tilde{\Omega}_{k}+q_{0} \zeta_{i j k}^{\psi} u_{j k}, \\
h_{i}^{p}= & q_{0} L_{2}(\nabla \times \hat{\boldsymbol{p}})_{j} \delta_{i j}^{\perp}+q_{0} \tau_{\Omega} \hat{p}_{j} \hat{p}_{r} \epsilon_{k i r} \nabla_{j} \tilde{\Omega}_{k} \\
& +q_{0} \hat{p}_{k} \epsilon_{k j i}\left(\tau_{c} \nabla_{j} c+\tau_{\sigma} \nabla_{j} \sigma+\tau_{\rho} \nabla_{j} \rho+\tau_{P} \nabla_{j} P\right) \\
& +q_{0} \tau_{k l}^{u} \hat{p}_{r} \epsilon_{r j i} \nabla_{j} u_{k l}+K_{7} h_{i}^{p} .
\end{aligned}
$$

Now we turn to a discussion of the dynamic chiral contributions. Using the condition $R=0$ and the general symmetry arguments outlined above, we obtain the following expressions for the chiral parts of the reversible currents up to linear order in the thermodynamic forces

$$
\begin{aligned}
j_{i}^{\sigma R} & =q_{0} \chi_{i j k}^{\sigma} A_{j k}, \\
j_{i}^{c R} & =q_{0} \chi_{i j k}^{c} A_{j k}, \\
X_{i j}^{u R} & =0, \\
j_{i}^{\mathrm{el} R} & =q_{0} \chi_{i j k}^{e} A_{j k}, \\
\sigma_{i j}^{R} & =-q_{0}\left(\chi_{k i j}^{c} \nabla_{k} \mu_{c}+\chi_{k i j}^{e} E_{k}+\chi_{k i j}^{\sigma} \nabla_{k} T\right), \\
X^{R} & =0, \\
Y_{i}^{R} & =0, \\
Y_{i}^{\Omega R} & =0,
\end{aligned}
$$

where we have disregarded gradients of the thermodynamic forces.

The material tensors $\chi_{i j k}^{\xi}$ describe purely chiral effects and contain one phenomenological parameter each (where $\xi \in \sigma, c, e)$

$$
\chi_{i j k}^{\xi}=\chi^{\xi}\left(\epsilon_{i k m} \hat{p}_{j} \hat{p}_{m}+\epsilon_{i j m} \hat{p}_{k} \hat{p}_{m}\right),
$$

totaling three purely chiral reversible transport parameters.

The contributions associated with $\chi_{i j k}^{\xi}$ couple reversibly extensional flows to heat, concentration and electric currents. All these contributions are absent in ordinary nematics and nematic gels and elastomers due to the lack of broken parity symmetry in the latter classes of materials. But we emphasize that they also exist in cholesteric low molecular weight materials as well as in cholesteric gels and elastomers. 
The dissipative part of the dynamics for polar nematic gels and elastomers, associated with the presence of the pseudoscalar $q_{0}$, can be discussed most succinctly in terms of the dissipation function. For the dissipation function of chiral polar nematic gels we get in addition to eq. (46)

$$
\begin{aligned}
R_{L}= & q_{0} \epsilon_{i j k} \hat{p}_{j} h_{k}^{p}\left(\psi_{c} \nabla_{i} \mu_{c}+\psi_{\sigma} \nabla_{i} T+\psi_{e} E_{i}\right) \\
& +q_{0} \epsilon_{i j k} \hat{p}_{j} L_{k}^{\perp}\left(\psi_{c}^{\Omega} \nabla_{i} \mu_{c}+\psi_{\sigma}^{\Omega} \nabla_{i} T+\psi_{e}^{\Omega} E_{i}\right) \\
& +q_{0} \psi_{j k}\left(\chi_{i j k}^{c \psi} \nabla_{i} \mu_{c}+\chi_{i j k}^{\sigma \psi} \nabla_{i} T+\chi_{i j k}^{e \psi} E_{i}\right) .
\end{aligned}
$$

It contains all the dissipative Lehmann-type effects relating gradients of temperature and concentration, and electric fields to director rotations (first line) and relative rotations (second line). In contrast to the static case, where there is no coupling to an electric field [52], such a contribution arises naturally in the dissipation function. On the other hand, there is no such dissipative coupling to density gradients, since there is no dissipative current associated with density (due to mass conservation). The third line describes appropriate dissipative couplings to elastic deformations, not considered before for polar systems. The material tensors $\chi_{i j k}^{\xi \psi}$ with $\xi \in\{\sigma, c, e\}$ contain one dissipative transport parameter each and are of the form of eq. (108).

We stress that these dissipative contributions occur for all gels and elastomers with macroscopic chirality including cholesteric and chiral smectic gels and elastomers.

The chiral parts of the dissipative currents then read

$$
\begin{aligned}
j_{i}^{\sigma D} & =-q_{0}\left(\epsilon_{i j k} \hat{p}_{k}\left[\psi_{\sigma} h_{j}^{p}+\psi_{\sigma}^{\Omega} L_{j}^{\perp}\right]+\chi_{i j k}^{\sigma \psi} \psi_{j k}\right), \\
j_{i}^{c D} & =-q_{0}\left(\epsilon_{i j k} \hat{p}_{k}\left[\psi_{c} h_{j}^{p}+\psi_{c}^{\Omega} L_{j}^{\perp}\right]+\chi_{i j k}^{c \psi} \psi_{j k}\right), \\
j_{i}^{\mathrm{el} D} & =-q_{0}\left(\epsilon_{i j k} \hat{p}_{k}\left[\psi_{e} h_{j}^{p}+\psi_{e}^{\Omega} L_{j}^{\perp}\right]+\chi_{i j k}^{e \psi} \psi_{j k}\right), \\
\sigma_{i j}^{D} & =0, \\
Y_{i}^{D} & =q_{0} \epsilon_{k j i} \hat{p}_{j}\left(\psi_{c} \nabla_{k} \mu_{c}+\psi_{\sigma} \nabla_{k} T+\psi_{e} E_{k}\right), \\
Y_{i}^{\Omega D} & =q_{0} \epsilon_{k j i} \hat{p}_{j}\left(\psi_{c}^{\Omega} \nabla_{k} \mu_{c}+\psi_{\sigma}^{\Omega} \nabla_{k} T+\psi_{e}^{\Omega} E_{k}\right), \\
X_{i j}^{u D} & =q_{0}\left(\chi_{k j i}^{c \psi} \nabla_{k} \mu_{c}+\chi_{k j i}^{\sigma \psi} \nabla_{k} T+\chi_{k j i}^{e \psi} E_{K}\right),
\end{aligned}
$$

totaling 9 purely chiral dissipative transport parameters.

As in the reversible case we have refrained from discussing effects involving gradients of the thermodynamic forces. However, if we take into account, e.g. $\nabla_{i} W$, then $X^{D}$ acquires chiral contributions $\sim \nabla_{i} h_{k}^{p}, \sim \nabla_{j} L_{k}^{\perp}$, or $\sim \nabla_{j} \psi_{j k}$ leading to counter terms of the form $\sim \nabla_{k} W$.

\section{Summary and conclusions}

In this paper we have presented the macroscopic dynamic equations for polar nematic gels and elastomers. We find that in comparison to nematic gels and to low molecular weight polar nematic liquid crystals a number of new cross-coupling terms arise, which might lead to applications. These are due, in particular, to static and dissipative dynamic coupling effects. Statically strain fields and relative rotations couple to electric fields and spatial variations of the polar preferred direction. Dissipatively heat currents, concentration currents and electric currents couple to relative rotations as well as to the molecular field of the polar preferred direction.

Partial support of this work by H.R.B. and H.P. through the Schwerpunktsprogramm SPP 1681 "Feldgesteuerte PartikelMatrix-Wechselwirkungen: Erzeugung, skalenübergreifende Modellierung und Anwendung magnetischer Hybridmaterialien" of the Deutsche Forschungsgemeinschaft is gratefully acknowledged. D.S. acknowledges the support of the Slovenian Research Agency, Grants Nos. PI-0099, N1-0019, and J1-7435.

\section{Appendix A. Ginzburg-Landau description of the phase transition isotropic-ferroelectric in polar nematic gels}

For the Ginzburg-Landau energy associated with the isotropic-ferroelectric transition in gels and elastomers we find the free energy

$$
\begin{aligned}
\varepsilon_{\mathrm{GL}}= & \frac{1}{2} a_{1} \boldsymbol{P}^{2}+\frac{1}{4} a_{2}\left(\boldsymbol{P}^{2}\right)^{2}+\frac{1}{6} a_{3}\left(\boldsymbol{P}^{2}\right)^{3}-\boldsymbol{E} \cdot \boldsymbol{P} \\
& +\left(\psi_{1}^{\rho} \delta \rho+\psi_{1}^{\sigma} \delta \sigma+\psi_{1}^{c} \delta c+\psi_{1}^{t} u_{i i}\right) \boldsymbol{P}^{2} \\
& +\frac{1}{2} \psi_{1}^{u} u_{i j}^{\mathrm{dev}} P_{i} P_{j}+\left(d_{1}+d_{2} \boldsymbol{P}^{2}\right) \nabla_{i} P_{i} \\
& +\left(\psi_{2}^{\rho} \delta \rho+\psi_{2}^{\sigma} \delta \sigma+\psi_{2}^{c} \delta c+\psi_{2}^{t} u_{k k}\right) \nabla_{i} P_{i} \\
& +\frac{1}{2} \psi_{2}^{u} u_{i j}^{\mathrm{dev}}\left(\nabla_{i} P_{j}+\nabla_{j} P_{i}\right) \\
& +b_{1}\left(\nabla_{i} P_{i}\right)\left(\nabla_{j} P_{j}\right)+b_{2}\left(\nabla_{i} P_{j}\right)\left(\nabla_{i} P_{j}\right),
\end{aligned}
$$

where $P_{i}$ denotes the full polarization vector, which is assumed to vanish in the isotropic gel, and where $\delta \rho, \delta \sigma$ and $\delta c$ are variations of density, entropy density and concentration and $u_{i j}$ is the strain tensor, with $u_{i j}^{\mathrm{dev}}=u_{i j}-\frac{1}{3} \delta_{i j} u_{k k}$ its deviator. The external field $\boldsymbol{E}$ enters the free energy in the standard electrostatic way.

The first two terms describe a second order ferroelectric transition $\left(a_{2}>0\right)$ that takes place when $a_{1}$ changes sign, which is therefore, for thermotropic systems, written as $a_{1}=\alpha_{1}\left(T-T_{c}\right)$; for barotropic or lyotropic systems $T$ is replaced by $p$ or $c$, accordingly. For $T<T_{c}$ the equilibrium polarization is $P_{\text {eq }}^{2}=\left|a_{1}\right| / a_{2}$, while the orientation of $P_{i}$ is arbitrary. In case $a_{2}<0$ a (positive) term cubic in $\boldsymbol{P}^{2}$ has to be added and the transition becomes weakly first order. In eq. (A.1) we have therefore kept a sixth order term $\sim a_{3}$, since many paraelectric - ferroelectric transitions in solids are known to be weakly first order.

The external field induces a finite polarization for all temperatures which is oriented along the field direction. For $T>T_{c}$ the polarization is small and increases considerably when reaching $T<T_{c}$. There is, however, no longer a phase transition in an external field, when the underlying phase transition is second order without an external field. 
The second line contains contributions that effectively add to $a_{1}$ thereby shifting the critical temperature $T_{c}$, when an external pressure, temperature, or concentration change is applied. This already applies to low molecular weight systems, while the $\psi_{1}^{t}$ contributions describes a change in $T_{c}$ when the elastomer is compressed or swelled.

The contribution $\psi_{1}^{u} u_{i j}^{\mathrm{dev}} P_{i} P_{j}$ describes the (volumepreserving part of) electrostriction. It does not only influence the value of the equilibrium polarization $P_{0}$, but also its orientation. Assuming, e.g., an external 2-dimensional deformation, $u_{x y}=u_{y x}=S$, the energetic minimum without an electric field is obtained for a polarization in the shear plane with $P_{x}=\mp P_{y}$ (for $\psi_{1}^{u} S \gtrless 0$ ) and $P_{x}^{2}=P_{y}^{2}=P_{\mathrm{eq}}^{2}+\left|\psi_{1}^{u} S\right| / a_{2}$. For a 3-dimensional elongation (or axial elongation), $u_{x x}=u_{y y}=-(1 / 2) u_{z z}=L$, the polarization is either (for $\psi_{1}^{u} L>0$ ) along the z-direction with $P_{z}^{2}=P_{\mathrm{eq}}^{2}+2 \psi_{1}^{u} L / a_{2}$ or (for $\psi_{1}^{u} L<0$ ) arbitrarily in the x/y plane with $P_{x}^{2}+P_{y}^{2}=P_{\mathrm{eq}}^{2}+\left|\psi_{1}^{u} L\right| / a_{2}$.

We note that the influence of a coupling of the form $U u_{i j}^{\text {dev }} Q_{i j}$ has been investigated above the isotropicuniaxial nematic phase transition, which is always weakly first order, in liquid crystalline elastomers and gels. While de Gennes studied the influence of a uniaxial external compression of dilatation [63], the influence of an external symmetric shear stress has been considered in ref. [64].

If in addition to an external elastic deformation also an electric field is applied, whose orientation does not accidentally coincide with the preferred polarization direction set by the deformation, there is a competition. Generally the resulting orientation of the polarization will be different from that of the field, rendering the system biaxial. In the hydrodynamics presented in the main body of this paper we neglect the orienting effects of elastic deformations and assume a uniaxial system with the preferred direction $P_{i}$, parallel to an electric field, if present.

A peculiar feature of any isotropic to polar transition is the existence of a linear splay term, $d_{1} \nabla_{i} P_{i}$, in the Ginzburg-Landau energy. Linear gradient terms in the energy generally allow for inhomogeneous textures as the thermodynamic ground state. A well-known example is the linear twist term in the Frank energy for chiral nematic liquid crystals that leads to the helical director structure. Other examples occur in liquid crystal systems with additional tetrahedral order, where such linear gradient terms are responsible for possible ambidextrous helicity and ambidextrous chirality $[65,66]$, splay-bend textures [67], and co-rotating helices $[68,69]$.

The case of linear splay in polar transitions, however, is different and more complicated. Although a constant splay, $\nabla_{i} P_{i}=$ const, definitely lowers the gradient part of $\varepsilon_{\mathrm{GL}}$, it necessarily requires a space-dependent, reduced polarization $P$ that spoils the homogeneous part of $\varepsilon_{\mathrm{GL}}$. It is also impossible for constant splay to have a defect-free texture of $\hat{p}_{i}$. In addition, with splay (internal and external) surfaces acquire charges that interact via Coulomb interaction, further reducing the stability of splay phases. For a thorough discussion of the possibility and stability of polar phases with splay textures in two and three dimensions we refer to [3]. As a result, splay phases can only exist close to $T_{c}$ or for large systems. In the main part of this manuscript, we derive the hydrodynamics for the case of a homogeneous ground state without splay.

The $\psi_{2}^{\xi}$ contributions in line four of $\varepsilon_{\mathrm{GL}}$ add to the magnitude of the linear splay term, $d_{1}$, when an external pressure, temperature, or concentration change is applied, or the elastomer is compressed or swelled. The nonlinear $d_{2}$ contribution describes the polarization dependence of the magnitude of the linear splay term within the power expansion of a Ginzburg-Landau treatment. The consequences of the contribution $\sim d_{2}$ have been investigated near the paraelectric-ferroelectric phase transition in solids and it was shown that this term is becoming important in the connection with incommensurate phases [70]. We note that there is also a nonlinear gradient term of the form $\sim P_{i} \nabla_{i}\left(P_{j} P_{j}\right)$, which differs from the contribution $\sim d_{2}$ in eq. (A.1) only by a surface term.

For the ferroelectric transition in gels or elastomers, there is an additional, qualitatively different, linear gradient term, $\psi_{2}^{u} u_{i j}^{\mathrm{dev}}\left(\nabla_{i} P_{j}+\nabla_{j} P_{i}\right)$, if there is an external (traceless) elastic deformation. Since it is a linear gradient term, it promotes, like the linear splay term, inhomogeneous textures of the polarization. However, the possibilities are not restricted to splay textures, but depend on the topology of the inducing elastic deformations. In particular, 2-dimensional symmetric shear patterns or planar and 3-dimensional elongations lead to appropriate structures of the polarization. Of course, all these patterns have the drawback, that necessarily they cannot be defect-free and $P$ is not constant. Such induced structures can favorably interfere with the possible spontaneous splay structure, thus reducing the stability of the homogeneous state. They also can compete with the splay structure, allowing for phase transitions between a splay phase and another (induced) inhomogeneous pattern depending on the strength of the driving elastic deformation.

Open Access This is an open access article distributed under the terms of the Creative Commons Attribution License (http://creativecommons.org/licenses/by/4.0), which permits unrestricted use, distribution, and reproduction in any medium, provided the original work is properly cited.

\section{References}

1. H. Hsiung, Th. Rasing, Y.R. Shen, F.P. Shvartsman, I.R. Cabrera, V.A. Krongauz, J. Chem. Phys. 87, 3127 (1987).

2. H. Zimmermann, R. Poupko, Z. Luz, J. Billard, Z. Naturforsch. A 40, 149 (1985).

3. H. Pleiner, H.R. Brand, Europhys. Lett. 9, 243 (1989).

4. H.R. Brand, P.E. Cladis, H. Pleiner, Macromolecules 25, 7223 (1992).

5. T. Niori, T. Sekine, J. Watanabe, T. Furukawa, H. Takezoe, J. Mater. Chem. 6, 1231 (1996).

6. D.R. Link, G. Natale, R. Shao, J.E. McLennan, N.A. Clark, E. Körblova, D.M. Walba, Science 278, 1924 (1997). 
7. H.R. Brand, P.E. Cladis, H. Pleiner, Eur. Phys. J. B 6, 347 (1998).

8. H.R. Brand, P.E. Cladis, H. Pleiner, Eur. Phys. J. B 31, 147 (2003).

9. G. Pelzl, S. Diele, W. Weissflog, Adv. Mater. 11, 707 (1999).

10. T. Niori, J. Yamamoto, H. Yokoyama, Mol. Cryst. Liq. Cryst. 409, 475 (2004)

11. D. Shen, S. Diele, G. Pelzl, I. Wirth, C. Tschierske, J. Mater. Chem. 9, 661 (1999).

12. W. Weissflog, H. Nadasi, U. Dunemann, G. Pelzl, S. Diele, A. Eremin, H. Kresse, J. Mater. Chem. 11, 2748 (2001).

13. G. Pelzl, A. Eremin, S. Diele, H. Kresse, W. Weissflog, J. Mater. Chem. 12, 2591 (2002).

14. M. Hird, Y. Raoul, J.W. Goodby, H.F. Gleeson, Ferroelectrics 309, 95 (2004).

15. H.R. Brand, P.E. Cladis, H. Pleiner, Int. J. Eng. Sci. 38, 1099 (2000)

16. C.-C. Yen, M. Tokita, B. Park, H. Takezoe, J. Watanabe, Macromolecules 39, 1313 (2006).

17. M. Koike, C.-C. Yen, L. Yuqing, H. Tsuchiya, M. Tokita, S. Kawauchi, H. Takezoe, J. Watanabe, Macromolecules 40, 2524 (2007).

18. Y. Taguchi, C.-C. Yen, S. Kang, M. Tokita, J. Watanabe, J. Phys. Chem. B 113, 5341 (2009).

19. P. Martin, O. Parodi, P.S. Pershan, Phys. Rev. A 6, 2401 (1972).

20. D. Forster, Hydrodynamic Fluctuations, Broken Symmetry and Correlation Functions (Benjamin, Reading, MA, 1975).

21. H. Pleiner, H.R. Brand, in Pattern Formation in Liquid Crystals, edited by A. Buka, L. Kramer (Springer, New York, 1996) p. 15.

22. I.M. Khalatnikov, Introduction to the Theory of Superfluidity (Benjamin, New York, 1965).

23. M. Liu, Phys. Rev. Lett. 35, 1577 (1975).

24. H. Brand, M. Dorfle, R. Graham, Ann. Phys. (N.Y.) 119, 434 (1979).

25. M. Liu, Phys. Rev. A 19, 2090 (1979).

26. H.R. Brand, Phys. Rev. A 33, 643 (1986).

27. H.R. Brand, H. Pleiner, F. Ziebert, Phys. Rev. E 74, 021713 (2006).

28. H.R. Brand, H. Pleiner, Physica A 208, 359 (1994).

29. P.G. de Gennes, in Liquid Crystals of One- and TwoDimensional Order, edited by W. Helfrich, G. Heppke (Springer, New York, 1980) p. 231.

30. M.S. Green, A.V. Tobolsky, J. Chem. Phys. 14, 80 (1946).

31. R.G. Larson, Constitutive Equations for Polymer Melts and Solutions (Butterworths, Boston, 1988).

32. H.R. Brand, H. Pleiner, W. Renz, J. Phys. (Paris) 51, 1065 (1990).

33. H. Pleiner, H.R. Brand, Mol. Cryst. Liq. Cryst. 199, 407 (1991).

34. H. Temmen, H. Pleiner, M. Liu, H.R. Brand, Phys. Rev. Lett. 84, 3228 (2000).

35. H. Pleiner, M. Liu, H.R. Brand, Rheol. Acta 39, 560 (2000).

36. H. Temmen, H. Pleiner, M. Liu, H.R. Brand, Phys. Rev. Lett. 86, 745 (2001).

37. M. Grmela, Phys. Lett. A 296, 97 (2002).
38. H. Pleiner, M. Liu, H.R. Brand, Rheol. Acta 41, 375 (2002).

39. H. Pleiner, M. Liu, H.R. Brand, Rheol. Acta 43, 502 (2004).

40. H. Pleiner, M. Liu, H.R. Brand, in IMA Volume in Mathematics and its Applications 141, edited by M.-C.T. Calderer, E.M. Terentjev (Springer, Berlin, 2005) p. 99.

41. H.R. Brand, H. Pleiner, Phys. Rev. A 35, 3122 (1987).

42. J.L. Ericksen, Arch. Rat. Mech. Anal. 113, 97 (1991).

43. S.R. de Groot, P. Mazur, Nonequilibrium Thermodynamics (North Holland, Amsterdam, 1962).

44. P.G. de Gennes, J. Prost, The Physics of Liquid Crystals (Oxford University Press, Oxford, 1995).

45. H.R. Brand, K. Kawasaki, J. Phys. C 19, 937 (1986).

46. W.P. Mason, Physical Acoustics and the Properties of Solids (D. Van Nostrand, New York, 1958).

47. H.R. Brand, H. Pleiner, Macromol. Rapid Commun. 11, 607 (1990).

48. H.R. Brand, P.E. Cladis, H. Pleiner, Phys. Rev. E 79 032701 (2009).

49. M. Liu, Phys. Rev. Lett. 43, 1740 (1979).

50. S. Bohlius, H.R. Brand, H. Pleiner, Phys. Rev. E 70, 061411 (2004).

51. P.G. de Gennes, The Physics of Liquid Crystals (Clarendon Press, Oxford, 1975).

52. H.R. Brand, H. Pleiner, Phys. Rev. A 37, 2736 (1988).

53. H.R. Brand, H. Pleiner, in Encyclopedia of Materials: Science and Technology (Elsevier) 5, 1214 (2001) and Cholesteric Liquid Crystals: Flow Properties, Thermo- and Electromechanical Coupling, in Reference Module in Materials Science and Materials Engineering, edited by Saleem Hashmi (Elsevier, Oxford, 2016) pp. 1-8.

54. D. Svenšek, H. Pleiner, H.R. Brand, Phys. Rev. E 78, 021703 (2008).

55. H.R. Brand, H. Pleiner, D. Svenšek, Phys. Rev. E 88 024501 (2013).

56. H. Pleiner, H.R. Brand, EPL 89, 26003 (2010).

57. F.C. Frank, Disc. Faraday Soc. 25, 19 (1958).

58. H.R. Brand, A. Fink, H. Pleiner, Eur. Phys. J. E 38, 65 (2015).

59. S.T. Kim, H. Finkelmann, Makromol. Chem. Rapid Commun. 22, 429 (2001).

60. A. Komp, J. Rühe, H. Finkelmann, Makromol. Chem. Rapid Commun. 26, 813 (2005).

61. H.R. Brand, Makromol. Chem. Rapid Commun. 10, 441 (1989).

62. A.M. Menzel, H.R. Brand, J. Chem. Phys. 125, 194704 (2006).

63. P.G. de Gennes, C.R. Acad. Sci. (Paris) B 281, 101 (1975).

64. H.R. Brand, Makromol. Chem. Rapid Commun. 10, 57, 317 (1989).

65. H.R. Brand, H. Pleiner, Eur. Phys. J. E 31, 37 (2010).

66. H. Pleiner, H.R. Brand, Eur. Phys. J. E 37, 11 (2014).

67. H. Pleiner, P.E. Cladis, H.R. Brand, Eur. Phys. J. E 20, 257 (2006)

68. H.R. Brand, H. Pleiner, P.E. Cladis, Physica A 351, 189 (2005).

69. H. Pleiner, H.R. Brand, Braz. J. Phys. 46, 565 (2016).

70. J.W. Felix, D. Mukamel, R.M. Hornreich, Phys. Rev. Lett. 57, 2180 (1986). 